\title{
Medidas preventivas nas práticas de inserção e manipulação de cateter de hemodiálise: estudo observacional ${ }^{*}$
}

\author{
Preventive measures in the practices of hemodialysis \\ catheter's insertion and manipulation: observational study*
}

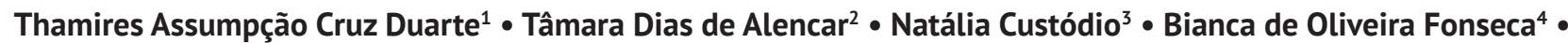 \\ Renata Flávia Abreu da Silva ${ }^{5}$
}

\begin{abstract}
RESUMO
Objetiva-se observar como as práticas de inserção e manipulação do cateter venoso central são realizadas em um serviço de Hemodiálise de um Hospital Federal do Rio de Janeiro. Estudo observacional de caráter descritivo de natureza quantitativa. Foram observados os procedimentos de inserção, manipulação e manutenção do cateter venoso central realizados por profissionais de saúde. Os resultados totalizaram 135 observações, das quais 16 foram de inserção e 119 de manipulação nos momentos pré e pós hemodiálise. No que tange à manipulação dos cateteres venosos centrais, 62 foram observados nos momentos pré e 57 nos momentos pós-hemodiálise. Entre as observações, $61 \%$ no período pré foram de conformidades e para os momentos pós, obteve-se $72 \%$ de conformidades. Conclui-se que as observações de não conformidades relacionadas às práticas de inserção e manipulação de cateter venoso central em hemodiálise podem ter apresentado este resultado por serem realizadas de forma não sistemática, o que pode colaborar com desfechos negativos relativos à diálise.
\end{abstract}

Palavras-chave: Infecções Relacionadas à Cateter; Cuidados de Enfermagem; Diálise Renal; Cateteres Venosos Centrais.

\begin{abstract}
The aim is to observe how the insertion and manipulation practices of the central venous catheter are performed in a hemodialysis service of a Federal Hospital in Rio de Janeiro. This observational and descriptive study of a quantitative nature. All the professionals that manipulate the catheter anyway where observed. 135 observations regarding the insertion and manipulation of the central venous catheter where made, of witch: 16 where about insertion, 135 about manipulation before hemodialysis and 119 from manipulation after hemodialysis. The results demonstrate that $61 \%$ practices in conformity and $39 \%$ of practices before hemodialysis non-conformities. About the practices after hemodialysis $72 \%$ where in conformity and $28 \%$ noncompliance. It concludes that this research allowed to apprehend that due to the practices of insertion and manipulation of central venous catheter in hemodialysis haven't systematically performed., nonconformities are likely to occur, with outcome to dialysis events.
\end{abstract}

Keywords: Catheter-Related Infections; Nursing Care; Renal Dialysis; Central Venous Catheters.

\footnotetext{
${ }^{1}$ Enfermeira. Especialista em Enfermagem em Clínica Médica e Cirúrgica em caráter de residência pela Universidade Federal do Estado do Rio de Janeiro. Especialista em Enfermagem Intensiva de Alta Complexidade pela Universidade Estácio de Sá, thamiresacduarte@yahoo.com.br.

${ }^{2}$ Enfermeira. Especialista em Enfermagem em Clínica Médica e Cirúrgica em caráter de residência pela Universidade Federal do Estado do Rio de Janeiro, alencartd@gmail.com. ${ }^{3}$ Enfermeira. Mestre em Enfermagem pela Universidade Federal de São Carlos. Especialista em Enfermagem em Clínica Médica e Cirúrgica em caráter de residência pela Universidade Federal do Estado do Rio de Janeiro, custodionati@gmail.com.

${ }^{4}$ Mestre em Microbiologia Média e Humana. Enfermeira do Serviço de Controle de Infecção Hospitalar do Hospital Federal da Lagoa, bianca.micro@gmail.com.

${ }^{5}$ Enfermeira. Doutora em Ciências pela Universidade Federal do Estado do Rio de Janeiro. Professora adjunta do Departamento de Enfermagem Médico Cirúrgica da Escola Alfredo Pinto (EEAP) da Universidade Federal do Estado do Rio de Janeiro, rflavia@me.com.

*Trabalho de Conclusão de Curso de Pós-graduação em Enfermagem em Clínica Médica e Cirúrgica nos moldes de Residência pela Universidade Federal do Estado do Rio de Janeiro (2017).

As autoras participaram igualmente na elaboração, construção, desenvolvimento e conclusão deste artigo.

As autoras declaram ausência de conflito de interesse.

Os autores declaram ausência de conflitos de interesses.
} 


\section{INTRODUÇÃO}

A Infecção Primária da Corrente Sanguínea (IPCS) associada à Cateter Venoso Central (CVC) ganha destaque, uma vez que, impacta no aumento da morbi-mortalidade de pacientes hospitalizados, aumento de dias de internação e por consequência aumento de gastos públicos extras. Neste ínterim, dados Norte-Americanos apontam para um custo adicional de US\$50.000,00 por evento adverso desta natureza ${ }^{1}$.

A IPCS caracteriza-se por infecções de consequências sistêmicas graves, bacteremia ou sepse, sem foco primário identificável ${ }^{1}$. Contudo, dados da Agência de Vigilância Sanitária (ANVISA) pontuam que mais da metade das bacteremias nosocomiais estejam associadas a algum cateter intravascular.

Neste sentido, no que tange às taxas de infecção relacionada aos pacientes em hemodiálise dos Estados Unidos da América estima-se o valor de 26 por 1000 pacientes-ano e mortalidade de 176 por 1000 pacientes$\mathrm{dia}^{2}$. Assim, esta temática ganha notoriedade mundial, visto que a maioria dos pacientes em diálise requerem um acesso vascular, o qual é passível de sofrer um evento adverso relacionado à assistência à saúde, suscitando possíveis morbi-mortalidade ${ }^{1}$.

Com isto, a National Healthcare Safety Network (NHSN) designou 3 eventos de diálise que devem ser monitorados sistematicamente, a saber: uso de antimicrobiano por via endovenosa, hemoculturas positivas e evidência de infecção do sítio de inserção do $\mathrm{CVC}^{3}$.

Com o objetivo de aprimorar o cuidado prestado pelos profissionais de saúde e promover a segurança do paciente O Institute of Healthcare Improvement (IHI), criou em 2001 o bundle. Definido como um pacote de medidas preventivas elaborado através de práticas baseadas em evidências, que visa promover a qualidade e segurança dos cuidados prestados, bem como a prevenção de eventos adversos e descrito, geralmente, de 3 a 5 medidas ${ }^{4}$. Este deve ser adotado na prática de inserção, manipulação e manutenção do CVC, consoante com a quinta meta internacional de segurança do paciente, a qual recomenda a redução do risco de infecções associadas ao atendimento em saúde ${ }^{5}$.

Estudo pioneiro realizado em Unidades de Terapia Intensiva do Estado de Michigan, Estados Unidos da América, mostrou a eficácia da aplicabilidade de um bundle com 5 medidas na introdução do CVC para a prevenção de IPCS relacionada à CVC. A adoção deste bundle reduziu expressivamente as taxas de infecção ${ }^{6}$, mostrando a efetividade do método aplicado, bem como a possibilidade de reduzir taxas de infecção com a adoção de pacotes de medidas preventivas.

Assim, diante da experiência vivenciada na residência em enfermagem, em um setor de hemodiálise, verificouse a ausência de um instrumento sistematizado e baseado em evidências científicas acerca das práticas de inserção, manipulação e manutenção dos CVCs, gerando possíveis não conformidades e consequente IPCS. Desta forma, surgiu o interesse pela temática com vistas a contribuir para a segurança do paciente.

Este estudo objetivou observar como as práticas de inserção e manipulação do CVC eram realizadas em um serviço de Hemodiálise de um Hospital Federal do Rio de Janeiro.

\section{MÉTODO}

Estudo observacional de caráter descritivo de natureza quantitativa, realizado em um setor de Hemodiálise, de um Hospital Federal do Rio de Janeiro, que também é instituição de ensino, composto por 7 leitos, sendo 1 de isolamento. Os pacientes são provenientes do ambulatório de nefrologia e das enfermarias. 0 setor de Hemodiálise também atende pacientes de setores fechados deste hospital, como Unidade de Terapia Intensiva (UTI), Unidade Coronariana (UCO) e setor de Emergência Interna. A coleta de dados foi realizada nos 4 espaços.

Os participantes da pesquisa foram os profissionais de saúde, sendo eles das categorias médica e de enfermagem. As técnicas da coleta de dados foram realizadas mediante Observação Simples e Não Participante.

A técnica de observação ganha destaque uma vez que permite ao observador perceber fatos diretamente e reduz a subjetividade que envolve todo o processo de investigação social. Pode ser utilizada como procedimento científico, à medida que: a) serve a um objetivo formulado de pesquisa; b) é sistematicamente planejada; c) é submetida à verificação e controles de validade e precisão ${ }^{7}$. Esta apresenta como principal vantagem, em relação a outras técnicas, a de que os fatos são percebidos diretamente, sem qualquer intermediação ${ }^{7}$.

As observações foram realizadas pelas próprias pesquisadoras, enfermeiras residentes, de dezembro de 2016 a janeiro de 2017, de segunda à sexta-feira no período diurno. As observações focaram nas práticas de inserção, manipulação e manutenção dos CVCs de duplo e triplo lúmen, utilizados para procedimentos de hemodiálise, administração de medicamentos, transfusão sanguínea e hidratação por via intravenosa.

As práticas foram observadas em dois momentos e mediante dois instrumentos de Pacotes de Medidas Preventivas (bundle). 0 primeiro bundle de inserção de CVC abrangeu a conduta de médicos preceptores e residentes, de acordo com os seguintes itens: degermação das mãos com clorexidina; uso de barreira máxima (capote estéril de mangas longas, gorro, máscara, luvas estéreis e campos estéreis (ongos); degermação da pele no sítio de inserção com clorexidina degermante $4 \%$ por 2 minutos; antissepsia da pele no sítio de inserção com clorexidina alcoólica; e dificuldade técnica durante o procedimento.

O segundo, de manipulações do CVC, foi observado nos momentos pré e pós HD e foi composto pelos seguintes itens: higienização das mãos antes de manipular o CVC; curativo com gaze estéril; curativo limpo, seco e bem fixado; 
curativo datado; desinfecção de conexões com álcool a 70\%; dispositivos de conexão protegidos; almotolias datadas e protegidas. Cabe destacar que as manipulações são de incumbência principal das (os) enfermeiras (os), das (os) enfermeiros (as) residentes, e auxiliares de enfermagem.

Para o processamento dos dados coletados foi realizada a análise descritiva simples, utilizando o programa Microsoft Excel 2010 por meio de tabelas e gráficos.

Em conformidade com a Resolução 466/12 e suas complementares sobre pesquisa com seres humanos, o presente projeto foi cadastrado na Plataforma Brasil sob o CAAE 60903416.0.0000.5285 e submetido ao Comitê de Ética em Pesquisa com Seres Humanos da instituição proponente, sendo aprovado sob o parecer de número 1.862.4448 . O Termo de Consentimento Livre e Esclarecido (TCLE) foi entregue imediatamente aos profissionais de saúde após a observação dos procedimentos, a fim de não influenciar a prática. Se houvesse o aceite em participar do estudo, o TCLE era assinado e os dados considerados.

\section{RESULTADOS E DISCUSSÃO}

Os resultados referentes à inserção e manipulação do CVC totalizaram $n=135$ observações, das quais $n=16$ foram de inserção e $n=119$ de manipulação nos momentos pré e pós HD. Destaca-se que todos os profissionais inseridos no setor da pesquisa em questão, estavam de acordo e aceitaram participar do estudo, com isso não houveram dados excluídos.

As práticas de inserção do CVC estão descritas na tabela 1 e observa-se que no que se refere ao item Degermação das Mãos, houve $94 \%$ ( $n=15)$ de conformidade. Contudo, a Higienização das Mãos não foi realizada em uma situação, diante da dificuldade técnica do médico residente que recebeu o apoio do preceptor, o qual não se utilizou da degermação das mãos antes de calçar a luva estéril, bem como da paramentação preconizada. A campanha da Organização Mundial da Saúde (OMS) reitera os cinco momentos para Higienização das Mãos com foco no cuidado do paciente com cateter venoso periférico, e reforça a importância da higiene das mãos (HM) antes de calçar luvas estéreis 9 .

No que se refere à degermação da pele, o tempo de 2 minutos no uso de clorexidina degermante $4 \%$ e a retirada com o soro fisiológico a $0,9 \%$, protocolados pela instituição, tiveram $100 \%(n=16)$ de não conformidade. Em contrapartida, estudo demonstrou que para se obter a máxima concentração de clorexidina na pele, esta deve ser mantida por pelo menos 1 minuto ${ }^{10}$.

Quanto ao sítio de escolha para a punção 63\% $(n=10)$ utilizaram a veia femoral e $38 \%(n=6)$ a veia jugular. Contudo, guidelines recomendam que o sítio de preferência seja em extremidades superiores, e quando em extremidades inferiores deve-se estudar constantemente a subida do cateter $^{11}$. Pode haver exceção diante da presença de traqueostomia, o que aumenta as chances de infecção em cateteres em veias jugulares, e em pacientes agudos com discrasias sanguíneas ${ }^{12}$.

Cabe destacar que a recomendação de antissepsia da pele com clorexidina alcoólica $0,5 \%$ foram realizadas na totalidade das observações. A clorexidina alcoólica 0,5\% é proposta por bundle proveniente de estudo internacional e pioneiro ${ }^{6}$, por ser mais efetiva em comparação a outros antissépticos, com nível de evidência máximo $(\mathrm{A} 1)^{1}$.

Quanto à Dificuldade técnica durante a inserção observou-se $81 \%(n=13)$ de não conformidade, caracterizada por mais de uma tentativa ou insucesso da punção. A literatura aponta que a experiência profissional é diretamente proporcional ao tempo de duração do procedimento e o número de tentativas de inserção ${ }^{13}$. Neste sentido, recomenda-se o emprego do ultrassom para a localização e confirmação da inserção como estratégia para redução de infecções ${ }^{14}$.

No que tange a manipulação dos CVCs, as observações totalizaram $n=119$, sendo $n=62$ nos momentos pré e $n=$ 57 nos momentos pós-hemodiálise. No momento pré-

Tabela 1. Observações das Práticas em Conformidades e Não Conformidades Referentes à Inserção do CVC, Rio de Janeiro, 2017.

\begin{tabular}{lcccc}
\hline Componentes & \multicolumn{2}{c}{ Conforme } & \multicolumn{2}{c}{ Não conforme } \\
& $\mathrm{n}$ & $\%$ & $\mathrm{n}$ & $\%$ \\
\hline Inserção de CVC & & & & \\
$\quad$ Degermação das mãos & 15 & $94 \%$ & 1 & $6 \%$ \\
$\quad$ Uso de barreira máxima durante a inserção & 15 & $94 \%$ & 1 & $6 \%$ \\
$\quad$ Degermação da pele com clorexidina degermante - 2 min & - & - & 16 & $100 \%$ \\
$\quad$ Antissepsia da pele com clorexidina alcoólica & 16 & $100 \%$ & - & - \\
$\quad$ Dificuldade durante o procedimento & 3 & $19 \%$ & 13 & $81 \%$ \\
$\quad$ Sítio de inserção veia jugular interna & 6 & $38 \%$ & 10 & $63 \%$ \\
\hline Total de observações & 55 & $57 \%$ & 41 & $43 \%$ \\
\hline
\end{tabular}

Fonte: dados da pesquisa. 
diálise foram observadas $63 \%$ de conformidades, enquanto nos momentos pós, obteve-se $75 \%$ de conformidades, de acordo com tabela 2 e 3 , respectivamente.

Destaca-se na tabela 2 a observação de $100 \%$ de não conformidade quanto à data presente no curativo, assim como $100 \%$ de conformidade sobre os itens Dispositivos Protegidos e Almotolias Datadas.

No momento pós-diálise, ganha destaque a não conformidade novamente do item Curativo datado. O mesmo cenário foi constatado em um estudo, no qual este procedimento também não era registrado, o que pode interferir na periodicidade das trocas do curativo ${ }^{15}$.

Referente à prática de realização do curativo houve o uso de filme transparente uma vez no momento pré-diálise, enquanto no pós todos os curativos foram realizados com gaze estéril. Quanto ao risco de adquirir IPCS, quando comparado ao curativo com filme transparente, estudos versam sobre esta ser também uma opção segura para a oclusão do óstio do cateter ${ }^{2}$.

Contudo, as coberturas utilizadas diferenciam-se quanto ao intervalo de trocas, o curativo de gaze estéril pode ser mantido até 48 horas, salvo na presença de sujidade, umidade, e não aderência ${ }^{16}$ o que na pesquisa foi confirmado, uma vez que o curativo com gaze foi trocado em cada procedimento de hemodiálise, o qual obedecia este intervalo. Enquanto, o curativo com filme transparente pode permanecer até 7 dias, desde que esteja íntegro².

Pertinente à Fixação, Limpeza e Umidade 23\% ( $n=14)$ apresentaram-se não íntegros no pré, em contrapartida no pós todos os curativos foram realizados conforme o preconizado pelo bundle nos três aspectos. Ressalta-se

Tabela 2. Observações referentes à Conformidades e Não Conformidades na Manipulação do CVC Pré-HD, Rio de Janeiro, 2017

\begin{tabular}{lcccc}
\hline Componentes & \multicolumn{2}{c}{ Conforme } & \multicolumn{2}{c}{ Não conforme } \\
\hline Manipulação do CVC pré-HD & $\mathrm{n}$ & $\%$ & $\mathrm{n}$ & $\%$ \\
\hline HM antes de manipular o CVC & 4 & $6 \%$ & 58 & $94 \%$ \\
Curativo com gaze estéril & 61 & $98 \%$ & 1 & $2 \%$ \\
Curativo limpo, seco e bem fixado & 48 & $77 \%$ & 14 & $23 \%$ \\
Curativo datado & - & - & 62 & $100 \%$ \\
Desinfecção das conexões com álcool 70\% & 18 & $29 \%$ & 44 & $71 \%$ \\
Dispositivos protegidos & 62 & $100 \%$ & - & - \\
Almotolias datadas & 62 & $100 \%$ & - & - \\
Almotolias protegidas & 58 & $94 \%$ & 4 & $6 \%$ \\
\hline Total de observações & 313 & $63 \%$ & 183 & $37 \%$ \\
\hline
\end{tabular}

Fonte: dados da pesquisa.

Tabela 3. Observações referentes à Conformidades e Não Conformidades na Manipulação do CVC Pós-HD, Rio de Janeiro, 2017

\begin{tabular}{lcccc}
\hline Componentes & \multicolumn{2}{c}{ Conforme } & \multicolumn{2}{c}{ Não conforme } \\
Manipulação do CVC pós-HD & $\mathrm{n}$ & $\%$ & $\mathrm{n}$ & $\%$ \\
\hline HM antes de manipular o CVC & 10 & $18 \%$ & 47 & $82 \%$ \\
Curativo com gaze estéril & 57 & $100 \%$ & - & - \\
Curativo limpo, seco e bem fixado & 57 & $100 \%$ & - & - \\
Curativo datado & - & - & 57 & $100 \%$ \\
Desinfecção das conexões com álcool 70\% & 51 & $89 \%$ & 6 & $11 \%$ \\
Dispositivos protegidos & 57 & $100 \%$ & - & - \\
Almotolias datadas & 57 & $100 \%$ & - & - \\
Almotolias protegidas & 55 & $96 \%$ & 2 & $4 \%$ \\
\hline Total de observações & 344 & $75 \%$ & 112 & $25 \%$ \\
\hline
\end{tabular}

Fonte: dados da pesquisa. 
a importância de uma fixação adequada do curativo, independente da cobertura, a fim de evitar que o óstio entre em contato com o ambiente externo, reduzindo o risco de contaminação ${ }^{17}$.

Apesar do item Curativo Datado ter apresentado a maior taxa de não conformidade, a não realização da Higienização das Mãos (HM) antes da manipulação do cateter, despontase como o de maior notoriedade, visto que é uma medida básica, com evidência máxima na prevenção de Infecção Relacionada à Assistência à Saúde (IRAS) ${ }^{18}$. Constitui meta 5 no programa de segurança do paciente e é exigida na Portaria vigente do Ministério da Saúde (MS ${ }^{19}$.

No estudo totalizaram $94 \%(n=58)$ de práticas de Higienização das Mãos em não conformidade no pré e $82 \%$ ( $n=47)$ no pós. 0 que corrobora com estudo observacional realizado com os profissionais de enfermagem, que constatou adesão de 39,9\% antes e após procedimentos realizados, indicando ainda que 17,2\% realizaram a técnica da higienização em conformidade e que o álcool a 70\% não foi utilizado ${ }^{20}$.

Cabe destacar que, o setor observado disponibiliza preparação alcoólica a $70 \%$ para fricção das mãos, obedecendo às normas estipuladas pela ANVISA as quais regulamentam que esta é a solução de escolha para higienização das mãos, bem como deve estar disponível à beira-leito do paciente, de forma que os profissionais não necessitem deixar o local de assistência para realizar tal procedimento ${ }^{21}$.

Ressalta-se que o método de observação apresenta limitações, porém acredita-se que o fato da entrega do TCLE ter sido após, pode ter contribuído para minimizar o efeito Hawthorne, no qual o profissional quando observado muda seu comportamento e adere práticas em consonância com o preconizado somente naquele momento ${ }^{22}$. No estudo, apesar de existirem profissionais residentes presentes no setor e atentando-se às práticas, esta foi incipiente. Assim, ressalta-se a necessidade da continuidade de estratégias educativas para a melhoria da adesão à $H M$, com envolvimento da instituição e lideranças dos serviços de saúde ${ }^{18}$.

0 item Desinfecções de Conexões com álcool a $70 \%$ aponta $71 \%(n=44)$ de não conformidade no pré e de $11 \%(n=6)$ no pós, tabelas 2 e 3 . Dados da literatura evidenciam a importância da desinfecção dos hubs seguindo técnicas assépticas com intuito de prevenir infecção ${ }^{15-16}$. O uso da solução de clorexidina alcoólica $0,5 \%$ desponta-se como mais indicado quando comparado ao álcool a 70\%, devido ao seu efeito residual, pertinente à fricção deve ser realizada no mínimo 15 segundos embora a literatura recomende este tempo, a instituição em questão recomenda a fricção com álcool a 70\% com 3 gazes distintas ${ }^{16}$.

Salienta-se ainda, a importância de manter as conexões dos cateteres protegidas com dispositivos visando a redução da colonização, que no estudo apresentou 100\% de conformidade nos dois momentos ${ }^{15-16}$. Por fim, os itens referentes as almotolias datadas e protegidas não representaram importância quanto a não conformidade.

\section{CONCLUSÃO}

A presente pesquisa permitiu contemplar que devido às práticas de inserção e manipulação de CVC em hemodiálise não serem realizadas sistematicamente, não conformidades são passíveis de acontecer, possibilitando desfechos negativos relacionados a eventos de diálise. As pesquisadoras, com resguarde às questões éticas, retornaram ao setor para apresentação dos resultados, bem como sugestão para chefia de enfermagem e médica, com o intuito de adotarem um instrumento de pacotes de medidas preventivas tanto na inserção como na manipulação de CVC.

Porém, o estudo apresentou algumas limitações, a saber, tempo reduzido de coleta de dados e observação de apenas um serviço de hemodiálise, não podendo os dados serem generalizados para outras populações de estudo. Sugerem-se novas pesquisas que explorem esta temática com maior profundidade e que comparem instituições onde há serviços de hemodiálise.

\section{REFERÊNCIAS}

1. Brasil. Ministério da Saúde. Agência Nacional de Vigilância Sanitária. Série Segurança do Paciente e Qualidade em Serviços de Saúde. Medidas de Prevenção de Infecção Relacionada à Assistência à Saúde. Brasília: MS; 2013.

2. Silva TNV, et al. Approach to prophylactic measures for central venous catheter-related infections in hemodialysis: a critical review. Hemodial Int. 2014; 18: 15-23.

3. Centers for Disease Control and Prevention (CDC). Reduction in central line-associated bloodstream infections among patients in intensive care units - Pennsylvania, April 2001-March 2005. MMWR Morb Mortal Wkly Rep. [Internet] 2005 [acesso em 17 Abri 2016]; 54: 1013-6. Disponível em: http://www.cdc.gov/ mmwr/preview/mmwrhtml/mm5440a2.htm.

4. Silva ACSS, Santos El. Articulação entre ensino e pesquisa no cuidado à saúde da criança: relato de experiência. Enferm. Atual [Internet]. 2016 [acesso em 15 Jan 2017]; 78: 61-65. Disponível em: http://inderme.com.br/revistas/revista_16.pdf.

5. Brasil. Ministério da Saúde. Portaria $n^{\circ}$. 529, de 1 de abril de 2013. Institui o Programa Nacional de Segurança do Paciente (PNSP) [Internet]. Diário Oficial da União 1 abr 2013 [acesso em 20 Abri 2016]. Disponível em: http://bvsms.saude.gov.br/ bvs/saudelegis/gm/2013/prt0529_01_04_2013.html.

6. Provonost P, Needham D, Berenholtz S, Sinopoli D, et al. An Intervention to Decrease Catheter-Related Bloodstream Infections in the ICU. N Engl J Med [Internet] 2006 [acesso 17 Abri 2016]; 355:2725-32. Disponível em: http://www.nejm. org/doi/full/10.1056/NEJMoa061115.

7. Gil AC. Métodos e técnicas de pesquisa social. 6.ed. São Paulo: Atlas; 2008.

8. Brasil. Conselho Nacional de Saúde. Resolução $n^{\circ} 466$ de 12 de dezembro de 2012. Diretrizes e normas regulamentadoras 
de pesquisa envolvendo seres humanos [Internet]. Brasília. 2012 [acesso 20 Abri. 2016]. Disponível em: http://conselho. saude.gov.br/resolucoes/2012/Reso466.pd

9. Worl Health Organization (WHO). Clean Care is Safer Care. My five moments for hand hygiene, Geneva. Focus on caring for a patient with a central venous catheter [Internet]. 2015 [acesso 20 Abri. 2016. Disponível em: http://www.who.int/ gpsc/5may/HH15_CentralCatheter_WEB_EN.pdf?ua=1.

10. Edmiston CE Jr, et al. Evidence for a Standardized Preadmission Showering Regimen to Achieve Maximal Antiseptic Skin Surface Concentrations of Chlorhexidine Gluconate, 4\%, in Surgical Patients. JAMA Surg [Internet]. 2015 [acesso 20 Abri.] 2016; 150(11): 1027-1033. Disponível em: https://www.ncbi. nlm.nih.gov/pubmed/26308490.

11. O'Grady NP, Alexander M, Burns LA, et al. Guidelines for the prevention of intravascular catheter-related infections. Am. J. Infect. Control [Internet]. 2011 [acesso 20 Abri 2016]; 39: S1-34. Disponível em: < http://www.ajicjournal.org/article/ S0196-6553(11)00085-X/pdf>.

12. Ferreira V. Acesso venoso central para hemodiálise: avaliação prospectiva da ocorrência de complicações [dissertação] Ribeirão Preto: Universidade de São Paulo [Internet]. 2005 [acesso em 10 Jan 2017]. Disponível em: http://www.bdpi.usp. br/single.php?_id=001457046

13. Pedrolo E, De Lazzari LSM, Oliveira GLR et al. Evidências para o Cuidado de Cateter Venoso Central de Curta Permanência: Revisão Integrativa. Rev enferm UFPE on line. [Internet]. 2013 [acesso em 10 Jan 2017]; 7(5): 4199-208. Disponível em: http://www.revista.ufpe.br/revistaenfermagem/index.php/ revista/article/view/3236/pdf_2625.

14. Algieri RD, Ferrante MS, Mérola C. et al. Infecciones Asociadas a Catéteres Venosos Centrales. Hosp Aeronáut Cent [Internet] 2012 [acesso em 10 Jan 2017]; 7(2): 127-132. Disponível em: http://www.hac.mil.ar/publicaciones/revismedic/0702/127132infeccionesasociadas.pdf.

15. Jardim JM, Lacerda RA, Soares NJD, Nunes BK. Avaliação das práticas de prevenção e controle de infecção da corrente sanguínea em um hospital governamental. Rev Esc Enferm USP [Internet]. 2013 [acesso em 07 Dez 2016]; 47(1): 3845. Disponível em: http://www.scielo.br/pdf/reeusp/v47n1/ a05v47n1.pdf.

16. Marschall J, Mermel LA, Mohamad F, Hadaway L et al. Strategies to prevent central line-associated bloodstream infections in acute care hospitals: 2014 update. Infect Control Hosp Epidemiol [Internet]. 2014 [acesso em 20 Jan 2017]; 35(7): 753-771. Disponível em: http://digitalcommons.wustl. edu/cgi/viewcontent.cgi?article $=4453$ \& context=open_ access_pubs.

17. França NG, Cruz I. Infection in central venous catheters: bandage with sterile gauze or transparent film? - Sistematic literature review. Journal of Specialized Nursing Care [Internet]. 2013 [acesso em 10 Dez 2016]; 6(1). Disponível em: http://www.uff.br/jsncare/index.php/jsncare/article/ view/2555.

18. Teixeira PA, Braga ALS, Andrade M, Santana PPC.A contribuição da educação no enfrentamento das infecções relacionadas à assistência à saúde: Revisão integrativa. Enferm. Atual [Internet]. 2015 [acesso em 08 Jan 2017]; 73:14-22. Disponível em: http://inderme.com.br/revistas/revista_11.pdf.
19. Brasil. Ministério da Saúde. Portaria n. 2.616, de 12 de maio de 1998. Dispõe sobre diretrizes e normas para a prevenção e o controle das infecções hospitalares [Internet]. Brasília; 1998 [acesso em 20 Jan 2017]. Disponível em: http://www20. anvisa.gov.br/segurancadopaciente/index.php/legislacao/ item/portaria-n-2-616-de-12-de-maio-de-1998.

20. Santos TCR, Roseira CE, Piai-Morais TH, Figueiredo RM. Higienização das mãos em ambiente hospitalar: uso de indicadores de conformidade. Rev Gaúcha Enferm. 2014 [acesso em 20 Jan 2017]; 35(1): 70-77. Disponível em: http:// seer.ufrgs.br/index.php/RevistaGauchadeEnfermagem/ article/view/40930.

21. Brasil. Ministério da Saúde. Agência Nacional de Vigilância Sanitária. Resolução de Diretoria Colegiada n. 42, de 25 de outubro de 2010. Dispõe sobre a obrigatoriedade de disponibilização de preparação alcoólica para fricção antisséptica das mãos [Internet]. Brasília; 2010 [acesso em 10 Jan 2017]. Disponível em: http://bvsms.saude.gov.br/bvs/ saudelegis/anvisa/2010/res0042_25_10_2010.html.

22. The Joint Commission. Measuring hand hygiene adherence: overcoming the challenges. EUA [Internet]. 2009 [acesso em 20 Jan 2017]. Disponível em: https://www.jointcommission. org/assets/1/18/hh_monograph.pdf. 